\title{
Radiografia quantitativa hepática de cães tranquilizados com acepromazina
}

\author{
Hepatic quantitative radiography in dogs with acepromazine
}

\author{
Bernardo Fernandes Lopes $^{I^{*}}$ Marilda Onghero Taffarel $^{\mathrm{I}}$ Mariana Lorenção Feitosa $^{\mathrm{I}}$ \\ Lorena Adão Vescovi Séllos Costa ${ }^{\mathrm{I}}$ Eduardo Raposo Monteiro ${ }^{\text {II }}$ Fabiano Séllos Costa ${ }^{\text {III }}$
}

RESUMO

O maleato de acepromazina é um dos tranquilizantes mais utilizados em medicina veterinária, podendo causar esplenomegalia, mas não é conhecida a possibilidade de ocorrência de hepatomegalia. Objetivou-se, com o presente estudo, utilizar a radiografia quantitativa com auxilio digital para determinar o tamanho e volume do figado de cães tranquilizados com acepromazina. Foram radiografados, na projeção lateral direita e ventrodorsal do fígado, nove cães hígidos, antes e após a administração intramuscular de maleato de acepromazina, na dose de 0,1mg $\mathrm{kg}^{-1}$ de peso. Foi possivel caracterizar aumento das dimensões hepáticas $(P<0,05)$ entre os diferentes momentos experimentais com forte correlação positiva entre as medidas corpóreas. A técnica radiográfica quantitativa empregada neste estudo caracterizou com precisão o aumento de tamanho e volume hepático nos cães tratados com acepromazina.

Palavras-chave: fenotiazinico, exame radiográfico, fígado, canino, hepatomegalia.

\section{ABSTRACT}

The acepromazine is one of the most commonly tranquilizer used in veterinary medicine and its use may cause splenomegaly. However it is not known if it can cause hepatomegaly. The aim of this study is to evaluate the quantitative radiography with digital assistance to determine the liver size and volume of dogs tranquilized with acepromazine. Nine healthy dogs were radiographed in the lateral right and ventral-dorsal liver, before and after intramuscular administration of acepromazine at a dose of $0.1 \mathrm{~kg}^{-1}$. It was possible to characterize the size and increase in liver size $(P<0.05)$ between the different experimental moments with a strong positive correlation between the measures liver assets. The quantitative radiographic technique used in this study accurately characterized the increase in size and volume of the liver in dogs treated with acepromazine.

Key words: phenothiazine, radiographic examination, liver, canine, hepatomegaly.

\section{INTRODUÇÃO}

O fígado possui papel fundamental em numerosos processos biológicos. Sua grande capacidade de reserva e de regeneração protege o organismo e garante a manutenção de processos biológicos importantes. No entanto, essa capacidade dificulta o diagnóstico das afecções hepáticas, pois há necessidade de grande comprometimento deste órgão para que haja alteração nas enzimas hepáticas. Por outro lado, o tamanho hepático pode variar rapidamente já que $15 \%$ do volume hepático são constituídos por leitos vasculares (CENTER, 2004).

Entre os métodos de imagem utilizados para avaliar o fígado, encontram-se o exame radiográfico, a ultrassonografia, a cintilografia nuclear, a tomografia computadorizada e a ressonância nuclear magnética (GODSHALK et al., 1998; RAPTOPOULOS et al., 1991; LIMAMOND et al., 2004; QAYYUM et al., 2005; TSUJIMOTO et al., 2006; KODAMA et al., 2007; CHOI

'Departamento de Medicina Veterinária, Centro de Ciências Agrárias (CCA), Universidade Federal do Espírito Santo (UFES), Alto Universitário, s/n, CP 16, 29500-000, Alegre, ES, Brasil. E-mail: bernardoflopes@hotmail.com. *Autor para correspondência.

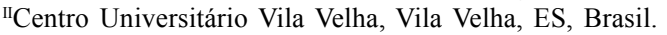

II'Departamento de Medicina Veterinária, Universidade Federal Rural de Pernambuco (UFRPE), Recife, PE, Brasil. 
et al., 2009; OLIVEIRA et al., 2009). A radiografia convencional rotineiramente é utilizada para avaliar as dimensões hepáticas em cães e gatos, sendo muitas vezes realizado um diagnóstico subjetivo de hepatomegalia ou microhepatia (FARROW, 2006). Com objetivo de obter resultados mais confiáveis, alguns estudos desenvolveram métodos para mensurar quantitativamente as dimensões hepáticas (VAN BREE et al., 1989; GODSHALK et al., 1989; GODSHALK et al., 1990) e, recentemente, esses métodos foram aprimorados com auxílio digital, através de réguas eletrônicas de programas computacionais que permitem eliminar a subjetividade e permitir maior reprodutibilidade interobservador (LARENTIS, 2005; ANDREA et al., 2007; CHOI et al., 2009; OLIVEIRA, et al., 2009).

Em diversas ocasiões, a tranquilização é necessária para a realização de exames de diagnóstico por imagem em cães. Desta maneira, é importante o conhecimento de alterações promovidas pelos fármacos anestésicos, a fim de evitar a presença de alterações iatrogênicas que podem interferir na interpretação dos achados imaginológicos (HALL \& WALTROUS, 2000).

A acepromazina é um agente da classe dos fenotiazínicos amplamente utilizado na tranquilização de cães. Os fármacos dessa classe produzem seu efeito por meio de antagonismo de receptores dopaminérigico no sistema nervoso central (BALDESSARINI \& TARAZI, 2001). Entretanto, esses fármacos não são seletivos para os receptores dopaminérgicos, interagindo também com receptores alfa-1 adrenérgicos periféricos. O bloqueio de receptores alfa-1 adrenérgicos vasculares resulta em vasodilatação periférica, redução na pressão arterial (MONTEIRO et al., 2007) e aumento da perfusão cutânea (BROCK, 1994) e visceral (O`BRIEN et al., 2004). Foi demonstrado também que a acepromazina reduz o hematócrito em cães, sugerindo o sequestro de células vermelhas no baço com consequente esplenomegalia (LANG et al., 1979; O‘BRIEN et al., 2004; MONTEIRO et al., 2007).

Por outro lado, existe pelo menos um estudo na literatura que relata a inexistência de correlação entre as alterações no hematócrito promovidas por agentes anestésicos (incluindo a acepromazina) e o volume do baço (WILSON et al., 2004), sugerindo o sequestro de células vermelhas em outros locais além do baço. Tendo em vista a grande capacidade vascular do fígado, formulou-se a hipótese que a administração da acepromazina resulta em aumento no volume hepático. O presente estudo teve como objetivo avaliar possíveis alterações induzidas pelo maleato de acepromazina nas dimensões hepáticas de cães, utilizando o exame radiográfico quantitativo com auxílio digital.

\section{MATERIAL E MÉTODO}

Foram avaliados nove cães adultos jovens, sem raça definida, sendo cinco machos e quatro fêmeas, com peso entre 5 e $15 \mathrm{~kg}$, provenientes do Centro de Controle de Zoonoses. Eles foram submetidos à avaliação clínica e laboratorial, envolvendo realização de hemograma, urinálise e dosagem dos níveis séricos de ALT, AST, FA, GGT, uréia e creatinina, a fim de submeter apenas animais saudáveis ao experimento. Posteriormente, foram alocados em baias coletivas e receberam água potável à vontade e ração comercial própria para a espécie.

Após período de quarentena e adaptação dos animais à manipulação pelos pesquisadores e às salas de exame, os cães foram pesados e submetidos à avaliação radiográfica após restrição hídrica e alimentar de 2 e 12 horas, respectivamente. Foram realizadas as projeções lateral direita e ventrodorsal com foco radiográfico sobre a décima costela e no pico expiratório. As radiografias foram realizadas imediatamente antes (M0) e 30 minutos após (M1) a administração do maleato de acepromazina (Acepran $0,2 \%$ - Vetnil) $(0,1 \mathrm{mg}$ $\mathrm{kg}^{-1}$ pela via intramuscular), conforme recomendado por BROCK (1994).

Para interpretação da imagem, as radiografias foram digitalizadas com câmera digital (Sony ${ }^{\circledR}$ Ciber-Shot DSC H50), com auxílio de tripé, e posteriormente avaliadas em programa de edição de imagem Adobe Photoshop CS4 ${ }^{\circledR}$ extended. Na projeção lateral, as variáveis avaliadas foram: 1) comprimento hepático $(\mathrm{CH})$, mensurado da extremidade mais cranial do diafragma, junto à inserção da veia cava, até a extremidade caudo-ventral do fígado (Figura 1);2) comprimento do corpo da décima primeira vértebra torácica (CT11); e 3) profundidade torácica (PT), traçando uma linha perpendicular da porção cranial da última esternébra até a borda ventral da vértebra torácica correspondente. Na projeção ventrodorsal, foi mensurada a largura do tórax (LT), traçando uma linha reta entre os recessos costofrênicos (Figura 2).

$\mathrm{O}$ cálculo do volume hepático $(\mathrm{VH})$ foi realizado pela fórmula 11,62+0,154 (CHxPTxLT), conforme descrito após análise de regressão por VAN BREE et al. (1989). Também foi avaliada a relação CH/ CT11 e a porcentagem de aumento hepático.

Para comparação dos valores obtidos nos diferentes momentos experimentais, foi realizada, inicialmente, a verificação da distribuição normal, pelo teste Shapiro Wilks e a avaliação subjetiva pela curva do histograma para auxiliar na escolha do teste estatístico. Devido à distribuição normal dos dados, foi escolhido o teste paramétrico $T$ para dados pareados 


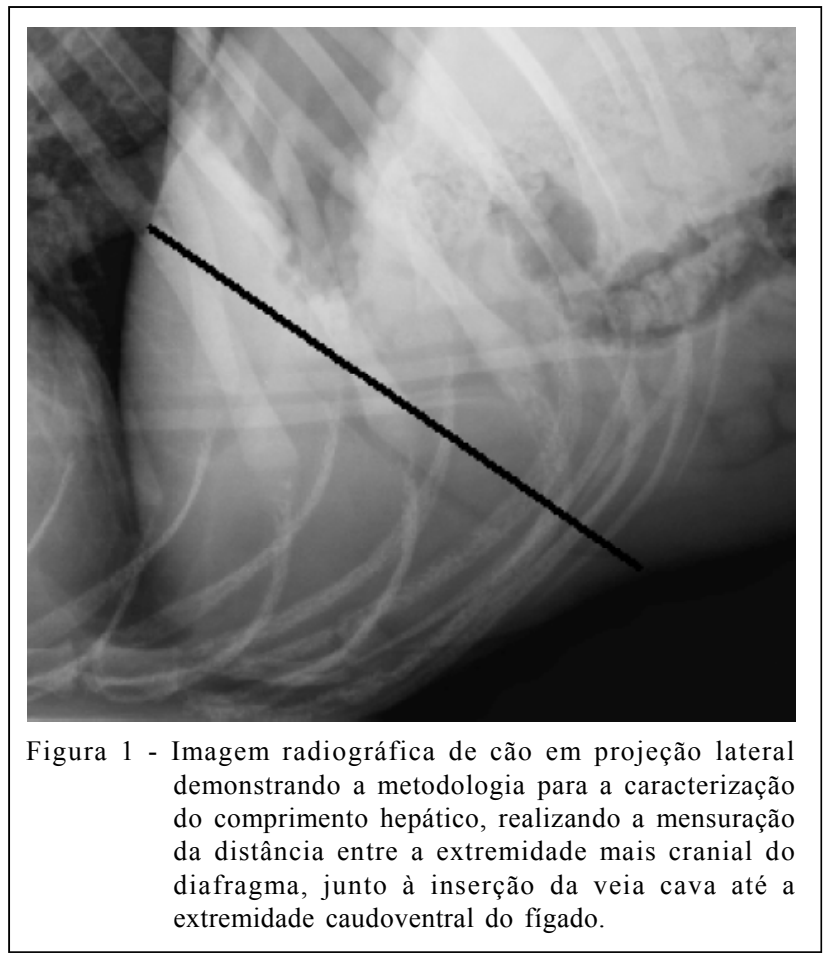

e coeficiente de correlação de Pearson para avaliar a correlação entre as variáveis estudadas.

\section{RESULTADOS E DISCUSSÃO}

A tabela 1 demonstra os valores médios e desvios padrão (DP) das variáveis estudadas antes e após a administração da acepromazina. Quando comparados os momentos, a fim de verificar possível aumento hepático promovido pela acepromazina, as variáveis comprimento hepático $(\mathrm{CH})$, volume hepático $(\mathrm{VH})$ e relação $\mathrm{CH} / \mathrm{CT} 11$ apresentaram valores maiores no momento $\mathrm{M} 1$ $(\mathrm{P}<0,05)$, com aumentos de $16,14 \pm 13,70 \%$; $15,24 \pm 9,53 \%$; e $13,1 \pm 14,2 \%$, respectivamente. Em estudo realizado por OLIVEIRA et al. (2009), a radiografia quantitativa foi eficiente para avaliar alterações no tamanho hepático promovidas pela administração de corticosteróides. De acordo com BROCK (1994), a acepromazina promove bloqueio dos adrenoreceptores alfa-1, resultando em aumento da perfusão visceral, causando esplenomegalia. Neste estudo, foi possível evidenciar o aumento do comprimento e volume hepático após a administração do maleato de acepromazina. Assim como ocorre no baço (LANG et al., 1979; O‘BRIEN et al., 2004), é possível que este aumento tenha ocorrido por vasodilatação em leitos vasculares hepáticos, resultado do bloqueio dos alfa-1 adrenoreceptores. Ressaltase que as variáveis profundidade torácica e largura torácica não variaram entre os momentos experimentais por não sofrerem ação do fármaco e pela padronização da fase do ciclo respiratório em que os exames radiográficos foram realizados.

A mensuração do comprimento hepático a partir da extremidade mais cranial do diafragma, junto à inserção da veia cava, à extremidade caudo-ventral do fígado também foi descrita por BREE et al. (1989), em pesquisa na qual concluíram que esta medida, assim

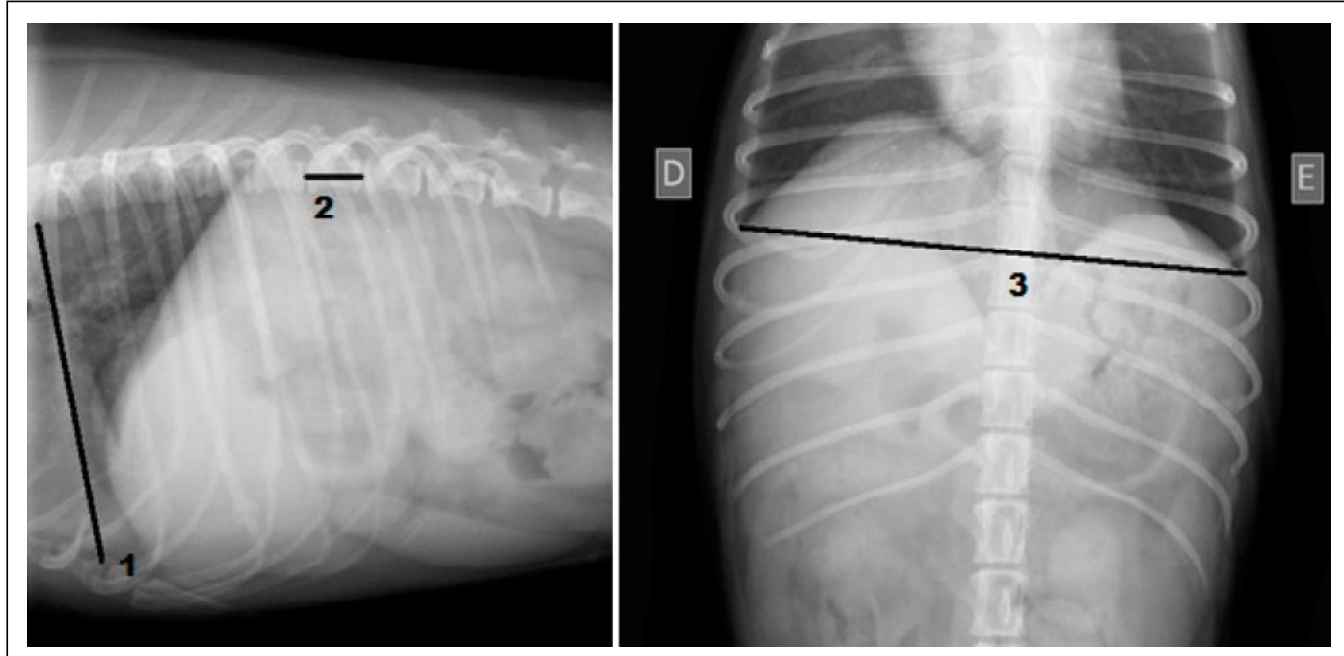

Figura 2 - Imagens radiográficas de cão em projeção lateral (imagem à esquerda) e ventrodorsal (imagem à direita), demonstrando a forma de aquisição de medidas corporais para obtenção do volume hepático e relação do comprimento hepático e tamanho vertebral. 1: profundidade torácica, 2: comprimento do corpo vertebral da décima primeira vértebra torácica, 3: largura torácica. 
Tabela 1 - Valores médios de medidas utilizadas para a avaliação do tamanho hepático \pm DP em nove cães antes (M0) e 30 minutos após a administração intramuscular de $0,1 \mathrm{mg} \mathrm{kg}^{-1}$ de acepromazina (M1). Os valores de $P$ são relatados (teste T pareado).

\begin{tabular}{llll}
\hline Variável & \multicolumn{1}{c}{ M0 } & M1 & P \\
\hline CT11 & $1,73 \pm 0,23^{\mathrm{a}}$ & $1,74 \pm 0,23^{\mathrm{a}}$ & 0,3615 \\
CH & $9,36 \pm 1,59^{\mathrm{a}}$ & $10,78 \pm 2,11^{\mathrm{b}}$ & 0,0014 \\
PT & $12,11 \pm 2,13^{\mathrm{a}}$ & $12,54 \pm 2,72^{\mathrm{a}}$ & 0,2731 \\
LT & $12,53 \pm 2,29^{\mathrm{a}}$ & $12,45 \pm 1,49^{\mathrm{a}}$ & 0,4310 \\
VH & $240,89 \pm 97,69^{\mathrm{a}}$ & $279,78 \pm 106,31^{\mathrm{b}}$ & 0,0029 \\
CH/CT11 & $5,4 \pm 0,8^{\mathrm{a}}$ & $6,1 \pm 0,7^{\mathrm{b}}$ & 0,0014 \\
\hline
\end{tabular}

Para análise dos resultados, deve-se considerar que letras iguais na mesma linha não diferem ao nível de 5\% de significância. CT11 comprimento do corpo vertebral da décima primeira vértebra torácica, $\mathrm{CH}$ - comprimento hepático, $\mathrm{PT}$ - profundidade torácica, LT - largura torácica, $\mathrm{VH}$ - volume hepático.

realizada, apresenta menor influência da fase respiratória do paciente. No atual estudo, verificou-se que a realização desta medida é de fácil execução embora não tenham sido comparadas diferenças entre fases respiratórias. Adicionalmente, foi possível verificar que a medida é sensível a pequenas alterações no comprimento hepático e, sendo assim, auxilia no diagnóstico de hepatomegalia, mesmo que em pequena proporção.

Houve forte correlação entre $\mathrm{VH}$ e o peso corpóreo e entre $\mathrm{VH}$ e CT11, dados que corroboram os achados de BREE et al. (1989). CHOI et al. (2009), utilizando a mesma fórmula, verificaram diferenças no volume hepático entre raças. Assim, foi possível comprovar a repetibilidade da medida, evidenciando a utilidade da radiografia quantitativa na avaliação do tamanho e volume do fígado, superando até mesmo a ultrassonografia, já que estudo realizado por GODSHALK et al. (1998) demonstrou a pobre correlação entre mensurações ultrassonográficas de dimensões hepáticas e volume real do fígado.

A relação $\mathrm{CH} / \mathrm{CT} 11$ apresentou média de 5,4 $\pm 0,8$ vértebras em $\mathrm{M} 0$ e 6,1 $\pm 0,7$ vértebras em $\mathrm{M} 1$. VAN BREE et al. (1989) descreveram uma relação CH/
CT11 de 5,5 $\pm 0,8$. CHOI et al. (2009) encontraram valores de $5,40 \pm 0,74$ para cães não braquicefálicos. WASHIZU et al. (2004) mensuraram a área hepática em radiografias digitalizadas de cães com e sem shunt portossistêmico e calcularam a relação da área hepática com comprimento da quarta vértebra lombar (CT4). Os autores verificaram que cães com shunt portossitêmico apresentam área hepática e relação área hepática/CT4 significativamente menor que cães saudáveis. Contudo, os autores não examinaram a correlação entre as variáveis. Neste estudo, escolheu-se a décima primeira vértebra torácica, baseado em estudos prévios (BREE et al., 1989) e devido ao fato de esta encontrar-se próxima ao foco radiográfico, minimizando distorções. Os resultados validam a relação comprimento hepático e comprimento vertebral como medida para mesurar o tamanho hepático normal, minimizando a interferência do peso e tamanho do indivíduo. Esta pequisa comprova ainda que a medida é sensível para diagnosticar pequenas alterações no tamanho do fígado em cães.

As tabelas $2 \mathrm{e} 3$ demonstram que houve forte correlação entre CH e CT11, resultado semelhante ao observado por CHOI et al (2009) e OLIVEIRA et al.

Tabela 2 - Coeficiente de correlação das variáveis mensuradas pelo exame de raios X quantitativo em nove cães no momento inicial (M0).

\begin{tabular}{lllllll}
\hline & CT11 & CH & PT & LT & VH & PESO \\
\hline CT11 & 1,00 & & & & \\
CH & $0,70^{*}$ & 1,00 & & & \\
PT & $0,93^{* *}$ & 0,47 & 1,00 & & & \\
LT & $0,71^{* *}$ & $0,70^{* *}$ & $0,60^{*}$ & 1,00 & & \\
VH & $0,82^{* *}$ & $0,83^{* *}$ & $0,80^{* *}$ & $0,90^{* *}$ & 1,00 & 1,00 \\
Peso & $0,88^{* *}$ & $0,68^{*}$ & $0,82^{* *}$ & $0,93^{* *}$ & $0,95^{* *}$ & \\
\hline
\end{tabular}

Para análise dos resultados, devem-se considerar correlações fortes, valores acima de 0,7 , e correlações moderadas, valores entre 0,5 e 0,7 . CT11 - comprimento do corpo vertebral da décima primeira vértebra torácica, $\mathrm{CH}$ - comprimento hepático, PT - profundidade torácica, LT - largura torácica, $\mathrm{VH}$ - volume hepático, ${ }^{*}$ Correlação moderada, ${ }^{* *}$ Correlação forte. 
Tabela 3 - Coeficiente de correlação das variáveis mensuradas pelo exame radiográfico quantitativo em nove cães 30 minutos após a administração intramuscular de $0,1 \mathrm{mg} \mathrm{kg}^{-1}$ de acepromazina (M1).

\begin{tabular}{lllllll}
\hline & CT11 & CH & PT & LT & VH & Peso \\
\hline CT11 & 1,00 & & & & \\
CH & $0,79^{* *}$ & 1,00 & & & \\
PT & $0,59^{*}$ & 0,41 & 1,00 & & \\
LT & $0,86^{* *}$ & $0,63^{*}$ & 0,28 & 1,00 & & \\
VH & $0,87^{* *}$ & $0,90^{* *}$ & $0,71^{* *}$ & $0,71^{* *}$ & 1,00 & 1,00 \\
Peso & $0,95^{* *}$ & $0,85^{* *}$ & 0,45 & $0,87^{* *}$ & $0,89^{* *}$ & \\
\hline
\end{tabular}

Para análise dos resultados, devem-se considerar correlações fortes, valores acima de 0,7 , e correlações moderadas, valores entre 0,5 e 0,7 . CT11 - comprimento do corpo vertebral da décima primeira vértebra torácica, $\mathrm{CH}$ - comprimento hepático, PT - profundidade torácica, LT - largura torácica, $\mathrm{VH}$ - volume hepático, ${ }^{*}$ Correlação moderada, ${ }^{* *}$ Correlação forte.

(2009). GODSHALK et al. (1990) encontraram alta correlação entre o peso hepático e a segunda vértebra lombar (L2), contudo os autores não analisaram a relação entre as medidas hepáticas e o comprimento de L2. As correlações existentes entre as medidas do comprimento hepático com o comprimento vertebral e volume hepático indicam que a utilização de apenas uma medida hepática, obtida em uma única projeção lateral, pode ser suficiente para estimar o tamanho hepático e apresenta sensibilidade com relação a alterações deste.

\section{CONCLUSÃO}

Nas condições do presente estudo, foi possível concluir que o maleato de acepromazina promove hepatomegalia. A radiografia quantitativa com auxilio digital possibilita mensuração precisa das dimensões hepáticas, facilitando a reprodutibilidade dos resultados. A relação do comprimento hepático/ vertebral permite a avaliação do tamanho hepático, minimizando a variação individual.

\section{COMITÊ DE ÉTICA E BIOSSEGURANÇA}

Este estudo foi realizado sob aprovação do Comitê de Ética e Bem-estar Animal da Universidade Federal do Espírito Santo, tendo sido respeitados todos os preceitos éticos de proteção aos animais.

\section{REFERÊNCIAS}

ANDREA, C.E. et al. Análise da morfometria nuclear: descrição da metodologia e o papel dos softwares de edição de imagem. Jornal Brasileiro de Patologia e Medicina Laboratorial, v.44, p.51-57, 2007. Disponível em: <http://www.scielo.br/ s c i e 1 o.p h p ? p i d = S 1667624442000880 $00100010 \&$ script $=$ sci_abstract $\&$ t $\operatorname{lng}=\mathrm{pt}>$. Acesso em: 14 out.2010. doi: 10.1590/S1676-24442008000100010.

BALDESSARINI, R.J.; TARAZI, F.I. Drugs and the treatment of psychiatrtic disorders: psychosis and mania. In: HARDMAN
J.G.; LIMBIRD, L.E. (Ed). Goodman \& Gilman's: the pharmacological basis of therapeutics. New York: McGrawHill; 2001. p.485-520.

BREE, H.V. et al. Radiographic assessment of liver volume in dogs. American Journal of Veterinary Research, v.50, n.9, p.1613-1615, 1989.

BROCK, N. Acepromazine revisited. Canadian Veterinary Journal, v.35, p.458-459, 1994. Disponível em: <http:// www.ncbi.nlm.nih.gov/pmc/articles/PMC1686255/pdf/ canvetj00356-0060.pdf $>$. Acesso em: 14 out.2010.

CENTER, S.A. Fisiopatologia, diagnóstico laboratorial e afecções do fígado. In: ETTINGER, S.J.; FELDMAN, E.C. Tratado de medicina interna veterinária: doenças do cão e do gato. 5.ed. Rio de Janeiro: Guanabara Koogan, 2004. p.1745-1816.

CHOI, J.H. et al. Radiographic evaluation of liver size in Pekingese dogs. In: CONGRESS OF THE IVRA, 15., 2009 Búzios, RJ. Final program of the $15^{\circ}$ congress of the IVRA. Búzios: IVRA, 2009. p.124. Disponível em: <http:// o n l i n e library.wiley.com/do i / $10.1111 /$ j . $1 \begin{array}{llllllllllllllllllllll} & 7 & 4 & 0 & 8 & 2 & 6 & 1 & . & 2 & 0 & 0 & 9 & . & 0 & 1 & 6 & 5 & 0 & . & \mathrm{x} & /\end{array}$ abstract? systemMessage $=$ Due + to + scheduled + mainten ance + access + to + the + Wiley + Online + Library + may + be + disrupte $\mathrm{d}+\mathrm{a} \mathrm{s}+\mathrm{fo} 11 \mathrm{ow} \mathrm{s} \% 3 \mathrm{~A}+\mathrm{S}$ a t u rd a y $\% 2 \mathrm{C}+16+\mathrm{O}$ ctober++New+York+0700+EDT+to+0900+EDT\%3B+London+ 1200+BST+to+1400+BST\%3B+Singapore+1900+SGT+to $+2100+$ SGT $>$. Acesso em: 15 out.2010. doi: 10.1111/j.17408261.2009.01650.x.

FARROW, C.S. Doença hepática. In: FARROW, C.S (Ed). Diagnóstico por imagem do cão e do gato. São Paulo: Roca, 2006. 748p.

GODSHALK, C.P. et al. Quantitative ultrasonic assessment of liver size in dog. Veterinary Radiology, v.29, n.4, p.162167, 1998. Disponível em: <http://onlinelibrary.wiley.com/ $\mathrm{doi} / 10.1111 / \mathrm{j} .17408261 .1988 . \mathrm{tb} 01769 . \mathrm{x} /$ abstract? systemMessage $=$ Due + to + scheduled + maintenance $+a$ ccess + to +the +Wiley+Online +Library+may+be+dis rupted + as + follows $\% 3 \mathrm{~A}+$ Saturday $\% 2 \mathrm{C}+16+$ October $++\mathrm{Ne}$ $\mathrm{w}+$ York $+0700+\mathrm{EDT}+\mathrm{to}+0900+\mathrm{EDT} \% 3 \mathrm{~B}+$ London+1 $200+\mathrm{BST}+$ to $+1400+\mathrm{BST} \% 3 \mathrm{~B}+$ Singapore $+1900+\mathrm{SGT}+$ to $+2100+$ SGT $>$. Acesso em: 14 out.2010. doi: 10.1111/j.17408261.1988.tb01769.x. 
GODSHALK, C.P. et al. Quantitative noninvasive assessment of liver size in clinical normal dogs. American Journal of Veterinary Research, v.51, n.9, p.1421-1426, 1990.

GODSHALK, C.P. et al. Nuclear scintigraphic assessment of liver size in clinically normal dogs. American Journal of Veterinary Research, v.50, n.5, p.645-650, 1989.

HALL, J.A.; WATROUS, B.J. Effects of pharmaceuticals on radiographic appearance of selected examinations of the abdomen and thorax. Veterinary Clinics of North America: Small Animal Practice, v.30, n.2, p.349-377, 2000.

KODAMA, Y. et al. Comparison of CT methods for determining the fat content of the liver. Annual Meeting of the American Roentgen Ray Society, v.188, p.1307-1312, 2007. Disponível em: <http://www.ajronline.org/cgi/content/abstract/ 188/5/1307>. Acesso em: 14 out. 2010. doi: 10.2214/ AJR.06.0992.

LANG, S.M. et al. Acetylpromazine administration: its effects on canine hematology. Veterinary Record, v.105, p.397$598,1979$.

LARENTIS, N.L. Avaliação da profundidade de lesões de cárie proximal por exames clínico, radiográfico com uso de filtro digital de cor e de secções histológicas digitalizadas em scanner. 2005. 75f. Dissertação de mestrado em odontologia - Curso de Pós-graduação em Odontologia, Universidade Federal do Rio Grande do Sul, RS.

LIMAMOND, P. et al. Macrovesicular hepatic steatosis in living related liver donors: correlation betwen ct and histologic findings. Radiology, v.230, n.1, p.276-280, 2004. Disponível em: < http://radiology.rsna.org/content/230/1/276.full>. Acesso em: 14 out. 2010. doi: 10.1148/radiol.2301021176.

MONTEIRO, E.R. et al. Effects of acepromazine on the cardiovascular actions of dopamine in anesthetized dogs. Veterinary Anaesthesia and Analgesia, v.34, p.312-321, 2007. Disponível em: <http://onlinelibrary.wiley.com/doi/ 10.1111/j.1467-2995.2006.00328.x/abstract>. Acesso em: 14 out. 2010. doi: 10.1111/j.1467-2995.2006.00328.x.

O'BRIEN, R.T. et al. Sonographic features of drug-induced splenic congestion. Veterinary Radiology \& Ultrasound, v.45, n.3, p.225-227, 2004. Disponível em: <http:// o n 1 in e 1 i b r a ry. wi ley.com/doi/ $10.1111 /$ j . $\begin{array}{lllllllllllllllllllll}1 & 7 & 4 & 0 & 8 & 2 & 6 & 1 & . & 2 & 0 & 0 & 4 & . & 0 & 4 & 0 & 3 & 9 & . & \mathrm{x}\end{array} /$ abstract? systemMessage $=$ Due + to + scheduled + maintenance + acce $\mathrm{ss}+$ to + the + Wiley + Online + Library + may + be + disrupted + as + follows $\% 3 \mathrm{~A}+$ Saturday $\% 2 \mathrm{C}+16+$ October ++ New + York +070 $0+\mathrm{EDT}+\mathrm{to}+0900+\mathrm{EDT} \% 3 \mathrm{~B}+\mathrm{London}+1200+\mathrm{B} \mathrm{S}$ $\mathrm{T}+$ to $+1400+\mathrm{BST} \% 3 \mathrm{~B}+$ Singapore $+1900+\mathrm{SGT}+$ to $+2100+\mathrm{SGT}$. . Acesso em: 14 out. 2010. doi; 10.1111/j.17408261.2004.04039.x.

OLIVEIRA, D.C. et al. Hepatic quantitative radiographic measures in cats treated with prednisolona. In: CONGRESS OF THE IVRA, 15., 2009, Búzios, RJ. Final program of the $15^{\circ}$ congress of the IVRA. Búzios: IVRA, 2009. p.69. Disponível em: $<\mathrm{http} / /$ onlinelibrary.wiley.com/doi/10.1111/j.17408261.1988.tb01769.x/ abstract? systemMessage $=$ Due + to + scheduled + maintenance + access + to + the + Wiley + Online + Library + may + be + disrupted + as + foll ow s $\% 3 \mathrm{~A}+\mathrm{S}$ at urday $\% 2 \mathrm{C}+16+\mathrm{October}++\mathrm{New}+$ York $+0700+\mathrm{EDT}+$ to $+0900+\mathrm{EDT} \% 3 \mathrm{~B}+$ London $+1200+\mathrm{BS}$ $\mathrm{T}+$ to $+1400+\mathrm{BST} \% 3 \mathrm{~B}+$ Singapore $+1900+\mathrm{SGT}+$ to $+2100+\mathrm{SGT}>$. Acesso em: 14 out. 2010. doi: 10.1111/j.1740-8261.2009.01650.x

QAYYUM, A. et al. Accuracy of liver fat quantification at MR imaging: comparasion of out-of-phase gradient-echo and fatsatured fast spin-echo techiniques-initial experience. Radiology, v.237, n.2, p.507-511, 2005. Disponível em: < http://radiology.rsna.org/content/237/2/507.abstract $>$. Acesso em: 14 out. 2010 . doi: 10.1148/radiol.2372040539.

RAPTOPOULOS, V. et al. Value of dual-energy CT in differentiating focal fatty infiltration of the liver from lowdensity masses. American Journal Radiology, v.157, p.721$725,1991$.

TSUJIMOTO, T. et al. Rapid onset of glycogen storage hepatomegaly in a type- 2 diabetic patient after a massive dose of long-acting insulin and large doses of glucose. Internal Medicine, v.45, n.7, p.469-473, 2006.

WASHIZU, M.A et al. An evaluation of radiographic hepatic size in dogs with portosystemic shunt. Journal of Veterinary Medicine and Science. v.66, n.8, p.977-978, 2004.

WILSON, D.V. et al. The effect of four anesthetic protocols on splenic size in dogs. Veterinary Anaesthesia and Analgesia, v.31, p.102-108, 2004. Disponível em: < http:// onlinelibrary.wiley.com/doi/10.1111/j. 1467 2987.2004.00152.x/full>. Acesso em 14 out. 2010. doi: 10.1111/j.1467-2987.2004.00152.x. 